\title{
Factores que determinan la elección de carrera profesional: en estudiantes de undécimo grado de colegios públicos y privados de Barrancabermeja*
}

Factors that determine the election of professional career: in students of eleventh grade of public and private schools of Barrancabermeja

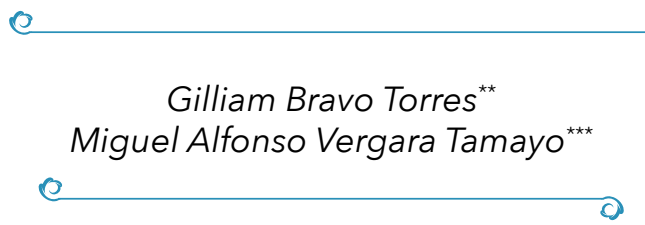

Recibido 12.01. $2018 \bullet$ Arbitrado 22. 01. $2018 \bullet$

Aprobado 15.02. 2018

* Este artículo es resultado de una investigación realizada con el objetivo de optar al título académico de Licenciada en Educación Básica con énfasis en Ciencias Sociales de la Universidad de Cundinamarca.

* Estudiante Licenciatura en Educación Básica con énfasis en Ciencias Sociales, Universidad de Cundinamarca, gilliambravo@hotmail.com

*** Docente - investigador del instituto universitario de la Paz, Zoot. Universidad de Cundinamarca, Fus a g s u gá / Cun d in a ma r c a Colombia, miguelvergara@msn.com

\section{Resumen}

Una de las decisiones más importante en la vida de una persona es la elección de su carrera profesional en la medida que esta definirá la vida que llevara al terminar la formación profesional, lo cual repercutirá directa e indirectamente en su vida familiar, laboral y social. Esta elección puede ser influenciada por factores como la familia, la economía, los intereses personales y medios masivos de comunicación. Dicho esto el propósito del presente estudio fue identificar y describir los factores que determinan la elección de carrera profesional de los estudiantes de grado undécimo de dos modalidades (públicos y privados) de seis colegios del municipio de Barrancabermeja / Santander. Se utilizó una metodología cuantitativa, corte trasversal y nivel descriptivo, con una muestra total de 225 estudiantes, se empleó como instrumento de recolección de información la encuesta diseñada para el presente estudio y se aplicó el consentimiento informado con los participantes. Los resultados muestran que los intereses personales de los estudiantes son el factor determinante al momento de la elección, tanto en los colegios públicos como en los privados, de ma- 
nera que prefieren la satisfacción que les genera ejecutar una tarea que le gusta, ante diversos factores externos como generar beneficios a la comunidad, el salario que devengaran, o la influencia familiar. Evidenciando, además, que no existe en la mayoría de los jóvenes la disposición de análisis o reflexión sobre la pertinencia de los diferentes factores ya que aunque algunos no influyan en la elección si representan gran importancia en los resultados de dicha decisión debido a los límites que estos pueden marcar.

Palabras claves: elección de carrera, educación media, profesión, futuro profesional, decisión.

\section{Abstract}

One of the decisions that are most important in the life of a person is the choice of his professional career to the extent that this will define the life that would lead to the end of the training, which will impact directly and indirectly in its familial, occupational and social life. This choice may be influenced by factors such as the family, the economy, personal interests and mass media. The purpose of the present study was to identify and describe the factors that determine the career choice of eleventh grade students in two modalities (public and private) of six schools in the municipality of Barrancabermeja/ Santander. Was used a quantitative methodology, transversal cutting and descriptive level, with a total sample of 225 students and as an instrument of data collection was used a survey designed for the present study and informed consent applied with participants. Obtaining as a result that the personal interests of the students, are the determining factor at the moment of said election, both in public and private schools, so they prefer the satisfaction that generates them to perform a task that they like, before diverse factors external as to generate benefits to the community, the salary that they will earn and the family influence. Proving further that there is no provision in the majority of young people for analysis or reflection on the relevance of the different factors since, although some do not influence the choice, they do represent great importance in the results of said decision due to the limits that these may mark.

Keywords: career choice, secondary education, profession, professional future, decision.

\section{Introducción}

La elección de la carrera profesional es una decisión que marcara el futuro de una persona y que, además, tiende a complejizarse en la medida en que 
no "siempre se lleva a cabo en función de la vocación de quien toma esta decisión, ya que en ésta (...), suelen intervenir ciertos factores, ya sean éstos internos o externos al sujeto que pueden llegar a influir" (Mendoza, 1994, p.10), de manera errónea o no, dependiendo del conocimientos que tengan los diferentes entes que intervienen en el proceso de dicha elección.

Esta decisión puede ser tomada a la ligera por muchos jóvenes y padres, a pesar de que ven en ello la posibilidad y el anhelo de mejorar su calidad de vida, basados generalmente en la concepción de la educación profesional como el camino para "surgir" o "llegar a ser alguien en la vida"; indicando así, que dicha elección será determinante para el futuro de los jóvenes, sumado a esto que "el estudiante tiene que definir su futuro e identidad ocupacional en plena crisis puberal y psicológica... debido a los cambios corporales, inseguridad, deseo de independencia, cambios continuos de interés entre otros" (Villada et al., 2002, p. 23). Además de tener que cargar con "las expectativas familiares y la variada información acerca de la oferta educativa que le llega a través de los medios" (Estrada, 2009 p. 1) de manera desproporcionada que termina por ejercer en el estudiante una presión que le dificulta la posibilidad de establecer los criterios adecuados para la elección de carrera profesional, como un debido proceso de planeación, investigación, reflexión y sin dejar de tener en cuenta el predominio de las habilidades y capacidades que se pueden reconocer en cada individuo.

Desde la experiencia como estudiante de pregrado y posteriormente como docente de la básica y media, surge a la vista un panorama preocupante respecto a los factores que se encuentran asociados a la elección de carrera profesional, tras la inseguridad que se hace evidente en los estudiantes de grados décimo y undécimo en lo referente a la elección de la carrera que estudiaran. Y cifras que según el M.E.N (2015) indican que:

para el nivel universitario el $46 \%$ de los estudiantes que ingresan en un periodo, abandonan sus estudios (...) confirmando que cerca de la mitad de los estudiantes que ingresan a los programas de educación superior se retiran sin culminar el proceso (p.2).

De tal manera el presente estudio se centró en la identificación de los factores determinantes que influyen en la elección de la carrera profesional de una muestra de estudiantes de Barrancabermeja que se encuentran cursando undécimo grado en tres colegios públicos y tres colegios privados. 


\section{Metodología}

Se realizo un estudio de enfoque cuantitativo, corte trasversal y nivel descritpivo. La muestra fue conformada por 225 estudiantes de Undecimo $\left(11^{\circ}\right)$ grado de Bachillerato, pertenecientes a seis (6) colegios de Barrancabermeja (3 colegios publicos y 3 colegios privados), los colegios publicos aportan a esta muestra un total de 137 estudiantes y los colegios privados aportan un total de 88. Se aplico una encuesta diseñada para el presente estudio, y sometida al juicio de investigadores expertos y ensayado previamente con una prueba piloto con un grupo de 20 estudiantes elegidos al azar. Para la aplicación de la encuesta se dio un tiempo de 30 minutos a los estudiantes. La aplicación del instrumento para la recopilación y el uso de informacion tuvo en cuenta las orientaciones éticas estipuladas por la reglamentacion legal para las investigaciones con los seres humanos.

\section{Resultados y discusión}

Prevalencia de factores de elección de carrera en los estudiantes de bachillerato de la muestra de colegios públicos y privados

Tabla N 1: Prevalencia de factores de elección de carrera en totalidad de la muestra de los estudiantes de bachillerato de colegios públicos y privados

\begin{tabular}{ccccc}
\hline $\begin{array}{c}\text { Intereses } \\
\text { personales }\end{array}$ & $\begin{array}{c}\text { Beneficios a } \\
\text { la sociedad }\end{array}$ & $\begin{array}{c}\text { Buen } \\
\text { salario }\end{array}$ & $\begin{array}{c}\text { Influencia } \\
\text { familair }\end{array}$ & $\begin{array}{c}\text { Lo que esta } \\
\text { de moda }\end{array}$ \\
\hline $0,42 \%$ & $0,32 \%$ & $0,22 \%$ & $0,04 \%$ & $0 \%$ \\
\hline
\end{tabular}

Los datos muestran que los intereses personales de los estudiantes son el factor predomínate en la elección de carrera, con un $42 \%$; seguido de la intención de generar beneficios a la sociedad con un $32 \%$; le sigue la posibilidad de optar a un buen salario con un $22 \%$ y finalmente esta la influencia familiar con un $4 \%$.

\section{Intereses personales de los estudiantes}

Los gustos o intereses se encuentran ligados a las "fuentes intrínsecas..., como la curiosidad, el interés por la tarea misma, la satisfacción de aprender y un sentimiento de triunfo. Es decir, la motivación intrínseca es aquella motivación asociada a actividades que son en sí su propia recompensa" (Cano, 2008, p7). 
Dicho esto, según el presente estudio los gustos o intereses de un estudiante son el factor predomínate en la elección de carrera con un $42 \%$ global;

De acuerdo a estos resultados los jóvenes anteponen sus gustos e intereses como el primer criterio a tener en cuenta para la elección de carrera, por encima de los otros, lo cual concuerda con las características que describen a la denominada Generación Y o Millennials que está conformada por "aquellos nacidos entre los años 1980 y 2000, es decir que actualmente tienen entre 10 y 30 años. (...) Los rasgos que presentan los jóvenes que integran esta generación son más individualistas que generaciones anteriores y reivindican la autonomía en sus opiniones y actuaciones, situando su ámbito personal por encima de consideraciones de orden laboral y social" (González, 2011, p 72). Dichos resultados sugieren, entonces, que estaríamos enfrentando la consolidación de una generación más orientada a velar por sus intereses sin tener la intención de detenerse a pensar en el bienestar común

\section{Generar beneficios a la comunidad}

Generar beneficios a la comunidad es el factor que ocupa el segundo lugar según el orden de importancia otorgado por los estudiantes con un $32 \%$ en total, cifras que se contraponen con los resultados del factor salarial.

Y aunque poco se ha estudiado este factor esto no es indicativo de que carezca de importancia frente al resto teniendo en cuenta que la elección de carrera repercutirá directa e indirectamente "en la realización personal, si, además, se consideran factores tan importantes como la vida que llevaremos al terminar la formación profesional, ello redundara en el beneficio de la familia y de la sociedad en general" (Avila, 2012, p. 26).

Y si dicho factor se perfila como uno de los que mayor predominio tienen en la elección de carrera de los jóvenes es una muestra de que si bien es cierto que de acuerdo a los resultados del presente estudio el individualismo o los intereses personales son lo que prevalece a la hora de planear su futuro y dar su primer paso, que es la elección de la carrera, también hay un alto número de jóvenes que están evidenciando el establecimiento de una conciencia social. Lo cual podría indicar que se le está abriendo paso a una lucha entre el bien individual y el bien común entre las nuevas generación de jóvenes que empiezan a comprender la conexión que existe entre sus vidas y el bienestar de otros con el mundo social y político y la importancia de participar para la creación de un mundo más justo pacífico y ecológico (Berman, 1997). 


\section{Buen salario}

Este factor se encuentra ligado de manera directa al factor económico el cual hace referencia en primer lugar a la situación económica de la familia, en cuanto sea capaz de asumir o no los gastos que implica la formación profesional, de la demanda que absorbe o no la mano de obra calificada y no calificada, de la remuneración que obtendrá el futuro profesional, como medio para mejorar el ingreso económico personal o de la familia (Cabrera, 1987).

Acceder a un buen salario determina la elección de carrea en un 22,22\% global.

"La necesidad de cubrir nuestras necesidades básicas y el hacer frente a un nivel de vida en alza creciente hace inevitable que al empezar un proceso de elección vocacional te preguntes qué tanta prioridad debes darle a este aspecto" (Sánchez, 2010, p. 6). Sin embargo, es preciso que el estudiante sepa que al enfocarse solo en la productividad de una carrera, se debe tener interés y habilidades para ejercer la actividad.

\section{Influencia familiar}

La elección de la carrera es una decisión que afecta a la familia tanto como esta influye en dicha decisión dado que el inicio de los hijos a "educación superior, parece ser una meta para todas las familias que cuentan con posibilidades de enfrentar este reto. Ostentar un título suele interpretarse como una garantía de éxito, prestigio, salario y poder avanzar en la estructura social" (Mendoza, 2000, p. 39).

En los jóvenes de las instituciones encuestadas la influencia familiar en la elección de carrera es de $4 \%$ global. Sin embargo, aunque resulta poco frecuente comentarios sobre desacuerdos de la familia con respecto a la carrera elegida por los hijos y pareciera que la familia no ejerce control en dicha elección, la realidad es que la influencia que ejercen cada vez se hace menos visible (Mendoza, 2000).

Lo que está de moda

Denota la carrera que por diversas razones, según el Diccionario de la Real Academia de la Lengua Española (2017), la moda es lo que "en un momento determinado goza de destacada aceptación", por lo general es producida por la influencia de los medios de comunicación y amigos.

Los medios de comunicación terminan siendo quienes educan "a los sujetos condicionando su forma de pensar y sentir, para tener una homogeneidad de pensamiento que no afecte los intereses mundiales y en específico del país" 
(Toquica, 2013, p. 69). "Todos estos medios de información difunden modelos estereotipados de lo que debe ser un joven actual. Le indica cómo vestirse, comportarse, relacionarse y hasta que carrera estudiar" (Conde, 2004, p.20).

Los medios de comunicación masivos, sumado a la valoración social de las profesiones puede generar efectos tales como como la distorsión de la información, trae consigo información errónea respecto a las carreras, ocupaciones, áreas de trabajo y planes de estudio (Avila, 2012).

También se puede decir que los amigos o grupos de amigos constituidos por aquellas personas con las que permanecen la mayor parte, influyen de manera destacada en las decisiones que toman los estudiantes. "Por lo que es posible que los miembros de un grupo de amigos, elijan...los estudios superiores, hasta en las mismas instituciones. Esto quiere decir que les interesa más seguir juntos en vez de la carrera que realmente les interesa" (Conde, 2004, p.18).

Los resultados arrojados por las encuestas indican que lo que está de moda no ejerce influencia alguna en los jóvenes tanto de las instituciones privadas como la publicas. Lo cual se podría considerar como un factor de riesgo menos al momento de la elección de carrera, debido a que "la elección de una carrera por moda... contribuye a una mala elección” (Conde, 2004, p. 19).

Prevalencia de factores de elección de carrera según la edad

Tabla N 2. Prevalencia de factores de elección de carrera según la edad

\begin{tabular}{ccccc}
\hline Edad & $\begin{array}{c}\text { Intereses } \\
\text { personales }\end{array}$ & $\begin{array}{c}\text { Beneficios a } \\
\text { la comunidad }\end{array}$ & $\begin{array}{c}\text { Buen } \\
\text { salario }\end{array}$ & $\begin{array}{c}\text { Influencia } \\
\text { familiar }\end{array}$ \\
\hline $15-16$ & $17,8 \%$ & $12,5 \%$ & $10,2 \%$ & $1,7 \%$ \\
$17-18$ & $20 \%$ & $16,4 \%$ & $13,9 \%$ & $2,2 \%$ \\
$19-20$ & $4,0 \%$ & $3,1 \%$ & $1,8 \%$ & $0,0 \%$ \\
\hline
\end{tabular}

Factores de elección de carrera profesional y edad del estudiante: De acuerdo a los resultados obtenidos $\left(\chi^{2}=7.559, g l=12\right)$ con un valor $\mathrm{p}=0.810$, no se rechaza la hipótesis nula, concluyendo que no hay relación entre la elección de carrera profesional y la edad del estudiante. 
Prevalencia de factores de elección de carrera según el sexo

Tabla $N$ 3. Prevalencia de factores que determinan la elección de carrera profesional en estudiantes de bachillerato según la variable de sexo

\begin{tabular}{cccccc}
\hline Sexo & $\begin{array}{c}\text { Intereses } \\
\text { personales }\end{array}$ & $\begin{array}{c}\text { Beneficios a } \\
\text { la sociedad }\end{array}$ & $\begin{array}{c}\text { Buen } \\
\text { salario }\end{array}$ & $\begin{array}{c}\text { Influencia } \\
\text { familair }\end{array}$ & $\begin{array}{c}\text { Lo que esta } \\
\text { de moda }\end{array}$ \\
\hline Femenino & $20,9 \%$ & $16,9 \%$ & $9,8 \%$ & $1,8 \%$ & $0 \%$ \\
masculino & $20,9 \%$ & $15,1 \%$ & $12,4 \%$ & $2,2 \%$ & $0 \%$ \\
\hline
\end{tabular}

Factores de elección de carrera profesional y género del estudiante: De acuerdo a los resultados obtenidos $\left(\chi^{2}=1.013, g l=3\right)$ con un valor $\mathrm{p}=0.798$, no se rechaza la hipótesis nula, concluyendo que no hay relación entre la elección de carrera profesional y el género masculino o femenino del estudiante.

Sin embargo, hay algunos valores de diferencia que aunque son mínimos vale la pena resaltar como el factor de Beneficios a la comunidad el cual es más alto en mujeres que en hombres, datos que concuerdan con otros estudios que indican la presencia de una mayor actitud prosocial en el género femenino (Plazas et al., 2010).

Prevalencia de factores de elección de carrera en los estudiantes de bachillerato de según el tipo de colegio: público y privado

Tabla N 4. Prevalencia de factores de elección de carrera según el tipo de colegio

\begin{tabular}{cccccc}
\hline $\begin{array}{c}\text { Tipo de } \\
\text { colegio }\end{array}$ & $\begin{array}{c}\text { Intereses } \\
\text { personales }\end{array}$ & $\begin{array}{c}\text { Beneficios a } \\
\text { la sociedad }\end{array}$ & $\begin{array}{c}\text { Buen } \\
\text { salario }\end{array}$ & $\begin{array}{c}\text { Influencia } \\
\text { familair }\end{array}$ & $\begin{array}{c}\text { Lo que esta } \\
\text { de moda }\end{array}$ \\
\hline publico & $22,7 \%$ & $15,6 \%$ & $17,8 \%$ & $1,3 \%$ & $0 \%$ \\
privado & $19,1 \%$ & $16,4 \%$ & $4,4 \%$ & $2,7 \%$ & $0 \%$ \\
\hline
\end{tabular}

Factores de elección de carrera profesional y tipo de colegio: De acuerdo a los resultados obtenidos $\left(\chi^{2}=15.224, g l=3\right)$ con un valor $\mathrm{p}=0.002$, se rechaza la hipótesis nula, concluyendo que hay relación entre la elección de carrera profesional y el tipo de colegio público o privado del estudiante.

Los intereses personales son el factor de prevalencia para la elección de carrera en los estudiantes de bachillerato con un $22,7 \%$ en públicos; $19,1 \%$ en privados. Tanto en colegios privados como en colegios públicos, este factor es el que predomina para la elección de carrera profesional, sin embargo, su influencia es mayor en los colegios privados. Generar beneficios a la comunidad es el segundo factor con 16,4\% en colegios privados y $15,6 \%$ en colegios 
públicos, sin embargo, el factor salarial supero dichos valores en los colegios públicos con un $17,8 \%$ y solo un $4,4 \%$ en los colegios privados.

De manera que en las instituciones privadas es mayor el interés por elegir la carrera pensando en ofrecer beneficios a la comunidad ante el deseo de obtener un buen salario. Mientras que en las instituciones públicas el factor salariar predomina sobre la opción de generar beneficios a la comunidad, evidenciando que la elección de carrera en colegios públicos está sujeto al predominio del factor socioeconómico. Esta interpretación se basa en la presunción de que en los colegios públicos predominan estudiantes con menor capacidad económica que los que estudian en colegios privados.

En cuanto a la influencia de familia se observó $2,7 \%$ en privados y $1,3 \%$ en públicos. Tanto en colegios púbicos como en los colegios privados la influencia que tiene la familia en los jóvenes es mínima.

\section{Conclusiones}

La elección de carrera determinara el presente y el futuro no solo de la persona sino también de su familia y del contexto social donde se desenvuelve, de manera que en su presente constituye una inversión de tiempo y dinero que indudablemente le puede sumar o restar a su condición socioeconómica y/o la de su familia; y en el futuro representará la calidad de vida en términos de comodidad, plenitud, felicidad, infelicidad o inconformidad, si a esto agregamos que "al comienzo de la planificación de la carrera profesional, a menudo tienen lugar consideraciones sobre la profesión en la que una persona se encuentra mejor" (Rauduvaitè y Lasauskienè, 2013, p. 175), además de repercutir en el entorno social de acuerdo al desempeño de su labor.

Entre los factores considerados para el presente estudio el que predomino como determinante para la elección de carrera fue el interés personal el cual que se encuentran ligado a las "fuentes intrínsecas..., como la curiosidad, el interés por la tarea misma, la satisfacción de aprender y un sentimiento de triunfo" (Cano, 2008, p. 7).

Si bien los estudiantes consideran que el factor económico es determinante en la elección de carrera, estos prefieren guiarse por sus intereses muchas veces sin tener en cuenta si poseen o no las capacidades o habilidades necesarias para desempeñar determinada profesión evidenciando de esta forma ciertos errores al momento de elegir. Al respecto Pineda (2015) dice: 
No se esperaría que estudiantes con bajos rendimientos en matemáticas durante el colegio, decidan estudiar programas de ingeniería, o estudiantes con bajos rendimientos en filosofía o lenguaje, decidan estudiar un programa de Ciencias Sociales y Humanas (p.3).

De lo anterior se puede observar que la falta de una completa orientación profesional permite que ocurra la consecuencia descrita en la cita anterior. Debido a que en la mayoría de los casos los orientadores no profundizan realmente en las vocaciones profesionales de los estudiantes de Bachillerato, y se dedican a realizar una orientación muy superficial basada en pruebas psicológicas que no dan espacio al desarrollo de proyectos educativos en diferentes ramas profesionales (administrativa, ambiental, salud, ingeniería, artística, entre otras) que permitan descubrir la verdadera vocación del estudiante.

Existen diferencias entre los jóvenes de los colegios públicos y privados. A pesar que en ambos prima la idea de elegir su carrera teniendo en cuenta principalmente sus intereses, en los primeros después de los intereses prima el factor económico apuntando a los beneficios económicas que recibirán al elegir determinada carrera, por otro lado, en los jóvenes de los colegios privados prevalece el deseo de generar beneficios a la comunidad antes que el salario que recibirán al ejercer su profesión. Concluyendo así que existe una estrecha relación entre los factores que consideran los jóvenes al elegir su carrera y el tipo de colegio (público o privado) teniendo en "cuenta que las características socioeconómicas de los estudiantes de los centros públicos y privados son distintas" (Giménez y Castro, 2017, p. 196), lo cual resulta determinante en la elección de carrera, dado que es más probable que, en promedio, los alumnos de centros privados provengan de familias con más recursos económicos y que los de centros públicos provengan de familias con ciertas limitaciones económicas. Corroborando de tal manera que la condición socioeconómica de los estudiantes juega también un papel determinante al momento de decidir por su futuro al elegir su carrera.

La influencia familiar parece no ejercer gran control en la elección de la carrera profesional de los hijos, ante esto se debe tener en cuenta que la influencia familiar no parte solo de un padre o madre que aconseja a su hijo la elección de una carrera que estos consideren que podrá asegurarle un buen futuro, sino que también puede ejercer control de formas menos evidentes como cuando un joven elige una carrera teniendo en cuenta la profesión de sus padres o hermanos y las condiciones que esta le genera, ya sea una difícil o buena situación económica. 
Evidentemente existen factores que pueden ejercer presión en los jóvenes que terminan por convertirse en una buena o mala elección, sin embargo, dicha elección es una decisión que no debería depender del nivel de presión que ejerza la familia, el medio social o económico debe entenderse como un aspecto propio de cada individuo; también es de gran importancia el conocimiento que tenga de sí mismo para descubrir la vocación y, finalmente, la necesidad de informarse acerca de la oferta de carreras y posibilidades de inserción laboral de estas (Secretaria de Educación para la Cultura, Medellín, 2008).

\section{Referencias}

Avila, V. (2012). Factores que influyen para la elección de carrera en los estudiantes de preparatoria (Doctoral dissertation, UPN-Ajusco). Tomado de http://200.23.113.51/pdf/28656.pdf

Berman, S. (1997). Children's social consciousness and the development of social responsibility. SUNY Press.

Cabrera, G. (1987). Factores externos que inciden en la elección profesional. Universidad de Antioquia. 1987. Tomado de http://ayura.udea.edu.co:8080/ jspui/bitstream/123456789/1000/1/CC0523.pdf

Cano, M. (2008). Motivación y elección de carrera. Revista Mexicana de Orientación Educativa, 5(13), 6-9, http://pepsic.bvsalud.org/scielo.php?script=sci_ abstract\&pid $=$ S1665-75272008000100003

Conde, C. (2004). La orientación vocacional y el proceso de elección de carrera. (Doctoral dissertation, UPN-Ajusco).Tomado de http://200.23.113.51/pdf/20067.pdf

Diccionario de la Real Academia de la Lengua Española. Actualización 2017. Tomado de http://dle.rae.es/

Estrada, (2009). Factores que intervienen en la elección de carrera de estudiantes de bachillerato de dos modalidades educativas. XI Congreso Nacional de Investigación Educativa / 16. Sujetos de la Educación / Ponencia Tomado de http://www. comie.org.mx/congreso/memoriaelectronica/v11/docs/area_16/2028.pdf

Giménez, G., Castro Aristizábal, G. (2017). ¿Por qué los estudiantes de colegios públicos y privados de Costa Rica obtienen distintos resultados académicos? Perfiles latinoamericanos, 25(49), 195-223, http://perfilesla.flacso.edu.mx/index.php/ perfilesla/article/view/841/771

González, R. S. (2011). La incorporación de la Generación Y al mercado laboral: el caso de una Entidad Financiera de la ciudad de Resistencia. Palermo Business 
Review, (5), 67-93. Tomado de http://www.palermo.edu/economicas/PDF_2011/ PBR5/5_Business04.pdf

Mendoza, M. (2000). Eleccion de carrera profesional: Visiones, promesas y desafios. Cambridge University Press.

Ministerio de Educación (2015). Estadísticas deserción y graduación. https://www. mineducacion.gov.co/sistemasdeinformacion/1735/articles-357549_recurso_3. pdf.

Pineda, L. (2015). Factores que afectan la elección de carrera: caso Bogotá (Masterss thesis).Tomado de http://cea.javeriana.edu.co/documents/153049/2786252/ Vol.+15_N3_Oct_2015.pdf/f47b2452-c9c0-4a60-886e-118076bd9936

Plazas, E.A., Morón Cotes, M.L., Santiago, A., Sarmiento, H., Ariza López, S.E., Patiño, C.D. (2010). Relaciones entre iguales, conducta prosocial y género desde la educación primaria hasta la universitaria en Colombia. Universitas Psychologica, 9 (2), 357-369, http://www.redalyc.org/articulo.oa?id=64716832005

Rauduvaitè, A., Lasauskienè, J. (2013). Factors Determining Personal Career Planning and Choices of Profession among Future Teachers of Music. Procedia-Social and Behavioral Sciences, 83, 175-179. Tomado de https://www.sciencedirect.com/ science/article/pii/S1877042813010641

Sánchez M. (2010). Elegir Carrera: Manual Express para el Alumno. Disponible en http://files.aula25.webnode.es/200000249-3b95a3c8fe/C\%C3\%93MO\%20 DECIDIR\%20UNA\%20CARRERA.docx.

Toquica, L. (2013). Valor que le atribuyen los estudiantes de grado noveno a undécimo al proyecto de orientación vocacional en el Instituto Pedagógico Nacional: análisis y descripción de sus características y dinámicas. Universidad Pedagogica Nacional. 2013. Tomado de http://repositorio.pedagogica.edu.co/xmlui/bitstream/ handle/123456789/210/TE-16009.pdf? sequence $=1$

Villada, C., López, L., Patiño, C., Ramírez, W., Jurado, C., Ossa, J. (2002). Factores Asociados a la Elección de Carrera y Universidad. Unipluri/versidad, 2, 23-30. Tomado de https://aprendeenlinea.udea.edu.co/revistas/index.php/unip/ article/viewFile/13163/11819 